ANNALES

POLONICI MATHEMATICI

$98.2(2010)$

\title{
On the oscillation of forced second order mixed-nonlinear elliptic equations
}

\author{
by Zhiting XU (Guangzhou)
}

\begin{abstract}
Oscillation theorems are established for forced second order mixed-nonlinear elliptic differential equations$$
\left\{\begin{array}{l}
\operatorname{div}\left(A(x)\|\nabla y\|^{p-1} \nabla y\right)+\left\langle b(x),\|\nabla y\|^{p-1} \nabla y\right\rangle+C(x, y)=e(x), \\
C(x, y)=c(x)|y|^{p-1} y+\sum_{i=1}^{m} c_{i}(x)|y|^{p_{i}-1} y
\end{array}\right.
$$

under quite general conditions. These results are extensions of the recent results of Sun and Wong, [J. Math. Anal. Appl. 334 (2007)] and Zheng, Wang and Han [Appl. Math. Lett. 22 (2009)] for forced second order ordinary differential equations with mixed nonlinearities, and include some known oscillation results in the literature.
\end{abstract}

1. Introduction and preliminaries. Since the pioneering work of Noussair and Swanson [14] and Picone [15, by using partial Riccati transformation [14] and Picone's identity [15], many researchers have studied the oscillation of half-linear partial differential equations with first order term

$$
\operatorname{div}\left(A(x)\|\nabla y\|^{p-1} \nabla y\right)+\left\langle b(x),\|\nabla y\|^{p-1} \nabla y\right\rangle+c(x)|y|^{p-1}=0 .
$$

We refer the reader to papers $[5,6,7,10,11,23,24,27,28,32]$, to monographs [12], 29], and to the references cited there. To the author's knowledge, very little is known about the oscillation of elliptic differential equations with forced terms. Recently, Jaroš, Kusano and Yoshida [5] and Zhuang [32] have established oscillation criteria for forced second order elliptic differential equations

$$
\operatorname{div}(A(x) \nabla y)+c(x)|y|^{\beta-1}=e(x), \quad \beta \geq 1,
$$

and $\mathrm{Xu}$ [25] has obtained Sun-Wong type oscillation theorems for forced second order damped elliptic equations with mixed nonlinearities

$$
\operatorname{div}(A(x) \nabla y)+\langle b(x), \nabla y\rangle+C(x, y)=e(x)
$$

2010 Mathematics Subject Classification: Primary 35B05; Secondary 35J15, 35J60.

Key words and phrases: oscillation, mixed-nonlinear, elliptic differential equations, second order, damped equation. 
with

$$
C(x, y)=q(x) y+\sum_{i=1}^{m} q_{i}(x)|y|^{\alpha_{i}-1} y,
$$

where $\alpha_{1}>\cdots>\alpha_{k}>1>\alpha_{k+1}>\cdots>\alpha_{m}>0$.

On the other hand, by using a Picone-type inequality, Jaroš, Kusano and Yoshida 6] have given oscillation criteria for half-linear partial differential equations with a forced term

$$
\operatorname{div}\left(a(x)\|\nabla y\|^{\alpha-1} \nabla y\right)+C(x)|y|^{\beta-1} y=e(x), \quad 0<\alpha<\beta,
$$

as well as the unforced elliptic equation with mixed nonlinearities

$$
\left.\operatorname{div}(a(x))\|\nabla y\|^{\alpha-1} \nabla y\right)+C(x)|y|^{\beta-1} y+D(x)|y|^{\gamma-1} y=0,
$$

where $0<\gamma<\alpha<\beta$. Furthermore, in [7] they have extended the results of [5] to elliptic differential equations with a first order term

$$
\operatorname{div}(A(x) \nabla y)+2\langle b(x), \nabla y\rangle+C(x)|y|^{\beta-1}=e(x), \quad \beta>1,
$$

and to unforced elliptic differential equations with mixed nonlinearities

$$
\operatorname{div}(A(x) \nabla y)+2\langle b(x), \nabla y\rangle+C(x)|y|^{\beta-1} y+D(x)|y|^{\gamma-1} y=0,
$$

where $0<\gamma<1<\beta$.

In this paper, we are concerned with the oscillation of forced second order mixed-nonlinear elliptic equations

$$
\left\{\begin{array}{l}
\operatorname{div}\left(A(x)\|\nabla y\|^{p-1} \nabla y\right)+\left\langle b(x),\|\nabla y\|^{p-1} \nabla y\right\rangle+C(x, y)=e(x), \\
C(x, y)=c(x)|y|^{p-1} y+\sum_{i=1}^{m} c_{i}(x)|y|^{p_{i}-1} y
\end{array}\right.
$$

where $p, p_{i}>0, i=1, \ldots, m, x=\left(x_{i}\right)_{i=1}^{N} \in \mathbb{R}^{N},\|x\|=\left[\sum_{i=1}^{N} x_{i}^{2}\right]^{1 / 2}, \nabla=$ $\left(\partial / \partial x_{i}\right)_{i=1}^{N}$, and $\langle$,$\rangle denotes the usual scalar product in \mathbb{R}^{N}$. We set $\Omega\left(r_{0}\right)=$ $\left\{x \in \mathbb{R}^{N}:\|x\| \geq r_{0}\right\}$ for $r_{0} \geq 0$.

Throughout this paper we shall also assume that

(A1) $A(x)=\left(a_{i j}(x)\right)_{N \times N}$ is an elliptic matrix with $a_{i j} \in \mathbf{C}_{\mathrm{loc}}^{1+\mu}\left(\Omega\left(r_{0}\right), \mathbb{R}\right)$ for all $i, j, \mu \in(0,1)$;

(A2) $b(x)=\left(b_{i}(x)\right)_{i=1}^{N}$ with $b_{i} \in \mathbf{C}_{\text {loc }}^{\mu}\left(\Omega\left(r_{0}\right), \mathbb{R}\right)$ for all $i$;

(A3) $e, c, c_{i} \in \mathbf{C}_{\mathrm{loc}}^{\mu}\left(\Omega\left(r_{0}\right), \mathbb{R}\right)$ for all $i$;

(A4) $p_{1}>\cdots>p_{k}>p>p_{k+1}>\cdots>p_{m}$;

or

(A5) $p_{1}>\cdots>p_{m}>p$.

A function $y \in \mathbf{C}_{\text {loc }}^{1+\mu}\left(\Omega\left(r_{0}\right), \mathbb{R}\right)$ with the property $a_{i j}\|\nabla y\|^{\alpha-1} \partial y / \partial x_{i} \in$ $\mathbf{C}_{\text {loc }}^{1+\mu}\left(\Omega\left(r_{0}\right), \mathbb{R}\right)$ for all $i, j$ is said to be a solution of (1.1) in $\Omega\left(r_{0}\right)$ if $y(x)$ satisfies (1.1) for all $x \in \Omega\left(r_{0}\right)$. For existence of solutions of (1.1), we refer the reader to the monograph [3]. We restrict our attention to nontrivial solutions 
$y(x)$ of (1.1), i.e., $\sup \{|y(x)|: x \in \Omega(r)\}>0$ for any $r \geq r_{0}$. A nontrivial solution $y(x)$ of $(1.1)$ is called oscillatory if the set $\left\{x \in \Omega\left(r_{0}\right): y(x)=0\right\}$ is unbounded; otherwise it is said to be nonoscillatory. Equation (1.1) is oscillatory if all of its solutions are oscillatory.

Consider the second order forced ordinary differential equation with mixed nonlinearities

$$
\left(p(t) x^{\prime}\right)^{\prime}+q(t) x+\sum_{i=1}^{m} q_{i}(t)|x|^{\alpha_{i}-1} x=e(t),
$$

where $p, q, q_{i}, e \in \mathbf{C}\left(\left[t_{0}, \infty\right), \mathbb{R}\right), p(t)$ is positive and differentiable, and $\alpha_{1}>$ $\cdots>\alpha_{k}>1>\alpha_{k+1}>\cdots>\alpha_{m}$. The oscillation of (1.2) was first studied by Sun and Wong [19] (see also [18]). Here our main objective is to extend the known oscillation criteria for (1.2) to (1.1). To motivate the formulation of our main results, we quote one of Sun-Wong's theorems for (1.2).

TheOREM 1.1 (19]). If for any $T \geq t_{0}$, there exist $a_{1}, b_{1}, a_{2}, b_{2}$ such that $T \leq a_{1}<b_{1} \leq a_{2}<b_{2}$ and

$$
\left\{\begin{array}{l}
q_{i}(t) \geq 0, \quad t \in\left[a_{1}, b_{1}\right] \cup\left[a_{2}, b_{2}\right], i=1, \ldots, m, \\
e(t) \leq 0, \quad t \in\left[a_{1}, b_{1}\right] ; \quad e(t) \geq 0, \quad t \in\left[a_{2}, b_{2}\right],
\end{array}\right.
$$

and if there exists a continuously differentiable function $u(t)$ such that $u\left(a_{i}\right)$ $=u\left(b_{i}\right)=0, u(t) \not \equiv 0$ for $t \in\left[a_{i}, b_{i}\right]$ and

$$
\int_{a_{i}}^{b_{i}}\left[Q(t) u^{2}(t)-u^{\prime 2}(t)\right] d t \geq 0 \quad \text { for } i=1,2,
$$

where

$$
Q(t)=k_{0}|e(t)|^{\eta_{0}} \prod_{i=1}^{n} q_{i}^{\eta_{i}}(t)+q(t)
$$

$k_{0}=\prod_{i=0}^{n} \eta_{i}^{-\eta_{i}}$, and $\eta_{0}, \eta_{1}, \ldots, \eta_{n}$ are positive constants satisfying conditions (a) and (b) of [19, Lemma 1], then equation (1.2) is oscillatory.

Theorem 1.1 was first given by Nasr [13] and Wong [22] for the case where $m=1$ and $q(t)=0$. In recent years, the Nasr-Wong theorem has been extended to various types of differential equations: see, for example, $[1,2,8,9,17,20,21,26,30-32]$. As pointed out in [19], it is interesting to study the oscillation of differential equations with mixed nonlinearities. In this paper, inspired by the ideas of Noussair and Swanson [14, Sun and Wong [19], and Zheng et al. [31, we shall establish some forced oscillation criteria for (1.1) which extend the results in [19, 30]. When $N=1$, our results include these of Sun and Wong [19] and Zheng et al. [31] for certain second order differential equations with mixed nonlinearities. Furthermore when $N \geq 2$, our results are more general than the oscillation criteria of 
Zhuang [32]. In particular, some interesting corollaries are established, and three examples of applications of our results are also included.

Before proceeding, we shall set some notations:

- $S(r)=\left\{x \in \mathbb{R}^{N}:\|x\|=r\right\}$,

- $\Omega[a, b]=\left\{x \in \mathbb{R}^{N}: a \leq\|x\| \leq b\right\}$,

- $\Omega(a, b)=\left\{x \in \mathbb{R}^{N}: a<\|x\|<b\right\}$,

- $A^{-1}(x)$ denotes the inverse of the matrix $A(x)$,

- $\nu(x)=x /|x|$ denotes the outside normal unit vector to the sphere $S(\|x\|)$,

- $d \sigma$ represents the integral element of $S(\|x\|)$,

- $\lambda_{\max }(x)$ and $\lambda_{\min }(x)$ denote respectively the largest and smallest eigenvalues of the matrix $A(x)$,

- $|A(x)|=\sup _{v \neq 0}\|A(x) v\| /\|v\|$ for any $v \in \mathbb{R}^{N}$ is the induced matrix norm.

Note that it follows from (A1) that $\lambda_{\max }(x) \geq \lambda_{\min }(x)>0$ and $|A(x)|=$ $\lambda_{\max }(x)$ for all $x \in \Omega\left(r_{0}\right)$. For any $[a, b] \subset\left[r_{0}, \infty\right)$, define

$$
\begin{aligned}
\mathcal{D}(a, b)=\left\{u \in \mathbf{C}^{1}[a, b], \mathbb{R}\right): u^{p+1}(r)>0 \text { for all } r \in(a, b), \\
\text { and } u(a)=u(b)=0\} .
\end{aligned}
$$

In order to prove our results we will need the following lemmas. The first one can be deduced from Lemma 2.1 of [19] using the transformation $\alpha_{i}=p_{i} / p$, the second one is the well-known arithmetic-geometric mean inequality [4, and Lemma 2.3 is a result on the maximal value of a function proved by a direct computation.

Lemma 1.1. Let $\left\{p_{i}\right\}, i=1, \ldots, m$, be an $m$-tuple satisfying (A4). Then there exists an m-tuple $\left(\eta_{1}, \ldots, \eta_{m}\right)$ with $\sum_{i=1}^{m} \eta_{i}<1$ and $0<\eta_{i}<1$ such that

$$
\sum_{i=1}^{m} p_{i} \eta_{i}=p .
$$

Lemma 1.2 ([4]). If $a_{i} \geq 0$ and $q_{i}>0$ with $\sum_{i=0}^{m} q_{i}=1$, then

$$
\sum_{i=0}^{m} q_{i} a_{i} \geq \prod_{i=0}^{m} a_{i}^{q_{i}} .
$$

Lemma 1.3. Let $a \geq 0, b>0$ and $X>0$. Then

(1) for $\gamma>p, a X^{\gamma}+b \geq \gamma p^{-p / \gamma}(\gamma-p)^{(p-\gamma) / \gamma} a^{p / \gamma} b^{(\gamma-p) / \gamma} X^{p}$;

(2) for $0<\gamma<p$, $a X^{\gamma}-b \leq \gamma p^{-p / \gamma}(p-\gamma)^{(p-\gamma) / \gamma} a^{p / \gamma} b^{(\gamma-p) / \gamma} X^{p}$.

2. Main results. We begin by extending Theorem 1.1 [19, Theorem 1]. 
Theorem 2.1. Let (A1)-(A4) hold. Assume that for any $r \geq r_{0}$, there exist $a_{1}, b_{1}, a_{2}, b_{2}$ such that $r \leq a_{1}<b_{1} \leq a_{2}<b_{2}$ and

$$
\begin{cases}c_{i}(x) \geq 0, & x \in \Omega\left[a_{1}, b_{1}\right] \cup \Omega\left[a_{2}, b_{2}\right], i=1, \ldots, m, \\ e(x) \leq 0, \quad x \in \Omega\left[a_{1}, b_{1}\right] ; \quad e(x) \geq 0, \quad x \in \Omega\left[a_{2}, b_{2}\right] .\end{cases}
$$

If there exist $u \in \mathcal{D}\left(a_{i}, b_{i}\right)$ and $\phi \in \mathbf{C}^{1}\left(\Omega\left(r_{0}\right), \mathbb{R}^{+}\right)$such that

$$
\int_{\Omega\left[a_{i}, b_{i}\right]} \phi(x)\left[C_{1}(x) u^{p+1}(\|x\|)-\frac{1}{(p+1)^{p+1}} \frac{|A(x)|^{p+1}}{\lambda_{\min }^{p}(x)}\|H(x)\|^{p+1}\right] d x>0
$$

for $i=1,2$, where

$$
H(x)=(p+1) u^{\prime}(\|x\|) \nu(x)-u(\|x\|)\left(b(x) A^{-1}(x)-\frac{\nabla \phi(x)}{\phi(x)}\right),
$$

and

$$
C_{1}(x)=c(x)+\left(\prod_{i=0}^{m} \eta_{i}^{-\eta_{i}}\right)|e(x)|^{\eta_{0}} \prod_{i=1}^{m} c_{i}^{\eta_{i}}(x),
$$

$\eta_{1}, \ldots, \eta_{m}$ are positive constants given in Lemma 1.1, and $0<\eta_{0}<1$ with $\sum_{i=0}^{m} \eta_{i}=1$, then equation (1.1) is oscillatory.

Proof. Suppose that $y=y(x)$ is a nonoscillatory solution of (1.1). Without loss of generality we may assume that $y(x)>0$ for all $x \in \Omega\left(a_{0}\right)$, $a_{0} \geq r_{0}$, where $a_{0}$ depends on the solution $y(x)$. When $y(x)$ is eventually negative, the proof is the same, using the annular domain $\Omega\left[a_{2}, b_{2}\right]$ instead of $\Omega\left[a_{1}, b_{1}\right]$. By the assumption, we can choose $a_{1}>b_{1} \geq a_{0}$ such that $c_{i}(x) \geq 0$ for all $i$ and $e(x) \leq 0$ for $x \in \Omega\left[a_{1}, b_{1}\right]$. Applying Lemma 1.2 to the case where

$$
u_{0}=\frac{1}{\eta_{0} y^{p}}|e(x)|, \quad u_{i}=\frac{1}{\eta_{i}} c_{i}(x) y^{p_{i}-p}, \quad i=1, \ldots, m,
$$

we observe that for $x \in \Omega\left(a_{0}\right)$,

$$
\begin{aligned}
&-e(x)+\sum_{i=1}^{m} c_{i}(x) y^{p_{i}}=|e(x)|+\sum_{i=1}^{m} c_{i}(x) y^{p_{i}} \\
&=y^{p}\left[\eta_{0} u_{0}+\cdots+\eta_{m} u_{m}\right] \geq y^{p}\left(\prod_{i=0}^{m} \eta_{i}^{-\eta_{i}}\right)|e(x)|^{\eta_{0}} \prod_{i=1}^{m} c_{i}^{\eta_{i}}(x) .
\end{aligned}
$$

Combining (2.3) and (1.1), we get

$$
\operatorname{div}\left(A(x)\|\nabla y\|^{p-1} \nabla y\right)+\left\langle b(x),\|\nabla y\|^{p-1} \nabla y\right\rangle+C_{1}(x)|y|^{p-1} y \leq 0 .
$$

Now, for $x \in \Omega\left(a_{0}\right)$, define

$$
w(x)=\frac{1}{y^{p}(x)}\left(A(x)\|\nabla y\|^{p-1} \nabla y\right) .
$$


From (1.1) it follows that

$$
\begin{aligned}
\operatorname{div} w(x)= & \frac{1}{y^{\alpha}} \operatorname{div}\left(A(x)\|\nabla y\|^{p-1} \nabla y\right)-\left\langle b(x) A^{-1}(x), w(x)\right\rangle \\
& -\frac{p}{y^{p+1}}\left\langle A(x)\|\nabla y\|^{p-1} \nabla y, \nabla y\right\rangle .
\end{aligned}
$$

Note that

$$
\|w(x)\| \leq|A(x)|(\|\nabla y\| / y)^{p}, \quad(\nabla y)^{T} A(x) \nabla y \geq \lambda_{\min }(x)\|\nabla y\|^{2} .
$$

Then, by (2.4)-(2.6),

$$
\operatorname{div} w(x) \leq-C_{1}(x)-\left\langle b(x) A^{-1}(x), w(x)\right\rangle-p \frac{\lambda_{\min }(x)}{|A(x)|^{q}}\|w(x)\|^{q},
$$

where $q=(p+1) / p$. Multiplying $(2.7)$ by $\phi(x)$, we get

$$
\begin{aligned}
\operatorname{div}(\phi(x) w(x)) \leq & -\phi(x) C_{1}(x)-\phi(x)\left\langle b(x) A^{-1}(x)-\frac{\nabla \phi(x)}{\phi(x)}, w(x)\right\rangle \\
& -p \frac{\phi(x) \lambda_{\min }(x)}{|A(x)|^{q}}\|w(x)\|^{q}
\end{aligned}
$$

Hence, multiplying both sides of $(2.8)$ by $u^{p+1}(\|x\|)$, we have

$$
\begin{aligned}
\int_{\Omega\left[a_{1}, b_{1}\right]} & \phi(x) C_{1}(x) u^{p+1}(\|x\|) d x \\
\leq & -\int_{\Omega\left[a_{1}, b_{1}\right]} u^{p+1}(\|x\|) \operatorname{div}(\phi(x) w(x)) d x \\
& -\int_{\Omega\left[a_{1}, b_{1}\right]} \phi(x) u^{p+1}(\|x\|)\left\langle b(x) A^{-1}(x)-\frac{\nabla \phi(x)}{\phi(x)}, w(x)\right\rangle d x \\
& -p \int_{\Omega\left[a_{1}, b_{1}\right]} \phi(x) u^{p+1}(\|x\|) \frac{\lambda_{\min }(x)}{|A(x)|^{q}}\|w(x)\|^{q} d x .
\end{aligned}
$$

By applying Green's formula to the first term of the right-hand side of (2.9), and noting that $u\left(a_{1}\right)=u\left(b_{1}\right)=0$, we have

$$
\begin{aligned}
\int_{\Omega\left[a_{1}, b_{1}\right]} u^{p+1}(\|x\|) & \operatorname{div}(\phi(x) w(x)) d x \\
& =\int_{a_{1}}^{b_{1}} u^{p+1}(r) \int_{S(r)} \operatorname{div}(\phi(x) w(x)) d \sigma d r \\
& =-(p+1) \int_{\Omega\left[a_{1}, b_{1}\right]} \phi(x) u^{p}(\|x\|) u^{\prime}(\|x\|)\langle\nu(x), w(x)\rangle d x .
\end{aligned}
$$


Combining this computation and (2.9), we get

$$
\begin{array}{r}
\int_{\Omega\left(a_{1}, b_{1}\right)} \phi(x) C_{1}(x) u^{p+1}(\|x\|) d x \leq \int_{\Omega\left(a_{1}, b_{1}\right)} \phi(x) u^{p}(\|x\|)\langle H(x), w(x)\rangle d x \\
-p \int_{\Omega\left[a_{1}, b_{1}\right]} \phi(x) u^{p+1}(\|x\|) \frac{\lambda_{\min }(x)}{|A(x)|^{q}}\|w(x)\|^{q} d x .
\end{array}
$$

By Young's inequality [4, Theorem 37],

$$
\begin{aligned}
u^{p}(\|x\|)\langle H(x), w(x)\rangle \leq & \frac{1}{(p+1)^{p+1}} \frac{|A(x)|^{p+1}}{\lambda_{\min }^{p}(x)}\|H(x)\|^{p+1} \\
& +p u^{p+1}(\|x\|) \frac{\lambda_{\min }(x)}{|A(x)|^{q}}\|w(x)\|^{q} .
\end{aligned}
$$

Substituting (2.11) into (2.10), we find that

$$
\begin{aligned}
\int_{\Omega\left[a_{1}, b_{1}\right]} \phi(x) C_{1}(x) u^{p+1}(\|x\|) d x & \\
\leq & \frac{1}{(p+1)^{p+1}} \int_{\Omega\left[a_{1}, b_{1}\right]} \phi(x) \frac{|A(x)|^{p+1}}{\lambda_{\min }^{p}(x)}\|H(x)\|^{p+1} d x,
\end{aligned}
$$

which contradicts (2.2).

If $c_{i}(x), i=k+1, \ldots, m$, is nonpositive on $\Omega\left[a_{1}, b_{1}\right] \cup \Omega\left[a_{2}, b_{2}\right]$, the following theorem is useful.

Theorem 2.2. Let (A1)-(A4) hold. Assume that for any $r \geq r_{0}$, there exist $a_{1}, b_{1}, a_{2}, b_{2}$ such that $r \leq a_{1}<b_{1} \leq a_{2}<b_{2}$ and

$$
\begin{cases}c_{i}(x) \geq 0, & x \in \Omega\left[a_{1}, b_{1}\right] \cup \Omega\left[a_{2}, b_{2}\right], i=1, \ldots, k, \\ e(x)<0, \quad x \in \Omega\left(a_{1}, b_{1}\right) ; \quad e(x)>0, \quad x \in \Omega\left(a_{2}, b_{2}\right) .\end{cases}
$$

If there exist $u \in \mathcal{D}\left(a_{i}, b_{i}\right), \phi \in \mathbf{C}^{1}\left(\Omega\left(r_{0}\right), \mathbb{R}^{+}\right)$and positive numbers $\delta_{i}$, $i=1, \ldots, k$, and $\varepsilon_{j}, j=k+1, \ldots, m$, with $\sum_{i=1}^{k} \delta_{i}+\sum_{j=k+1}^{m} \varepsilon_{j}=1$ such that

$$
\int_{\Omega\left[a_{i}, b_{i}\right]} \phi(x)\left[C_{2}(x) u^{p+1}(\|x\|)-\frac{1}{(p+1)^{p+1}} \frac{|A(x)|^{p+1}}{\lambda_{\min }^{p}(x)}\|H(x)\|^{p+1}\right] d x>0
$$

for $i=1,2$, where $H(x)$ is defined in Theorem 2.1, and

$$
\begin{aligned}
C_{2}(x)= & c(x)+\sum_{i=1}^{k} \alpha_{i}|e(x)|^{\left(p_{i}-p\right) / p_{i}} c_{i}^{p / p_{i}}(x) \\
& -\sum_{j=k+1}^{m} \alpha_{j}|e(x)|^{\left(p_{j}-p\right) / p_{j}} \bar{c}_{j}^{p / p_{j}}(x),
\end{aligned}
$$


with

$$
\begin{aligned}
\alpha_{i} & =p_{i} p^{-p / p_{i}}\left(p_{i}-p\right)^{\left(p-p_{i}\right) / p_{i}} \delta_{i}^{\left(p_{i}-p\right) / p_{i}}, & & i=1, \ldots, k, \\
\alpha_{j} & =p_{j} p^{-p / p_{i}}\left(p-p_{j}\right)^{\left(p-p_{j}\right) / p_{j}} \varepsilon_{j}^{\left(p_{j}-p\right) / p_{j}}, & & j=k+1, \ldots, m, \\
\bar{c}_{j}(x) & =\max \left\{-c_{j}(x), 0\right\}, & & j=k+1, \ldots, m,
\end{aligned}
$$

then equation (1.1) is oscillatory.

Proof. Assume to the contrary that $y=y(x)$ is a nonoscillatory solution and it is eventually positive, say $y(x)>0$ for all $x \in \Omega\left(a_{0}\right), a_{0} \geq r_{0}$. Note that (1.1) can be rewritten as

$$
\begin{aligned}
\operatorname{div}\left(A(x)\|\nabla y\|^{p-1} \nabla y\right)+\left\langle b(x),\|\nabla y\|^{p-1} \nabla y\right\rangle+c(x) y^{p} & \\
+\sum_{i=1}^{k}\left[q_{i}(x) y^{\alpha_{i}}-\delta_{i} e(x)\right]+\sum_{j=k+1}^{m}\left[q_{j}(x) y^{p_{j}}-\varepsilon_{j} e(x)\right] & =0 .
\end{aligned}
$$

By Lemma 1.3, for $x \in \Omega\left(a_{1}, b_{1}\right)$, we get

$$
\begin{aligned}
c_{i}(x) y^{p_{i}}-\delta_{i} e(x) & =c_{i}(x) y^{p_{i}}+\delta_{i}|e(x)| \\
& \geq \alpha_{i} c_{i}^{p / p_{i}}(x)|e(x)|^{\left(p_{i}-p\right) / p_{i}} y^{p}, \quad i=1, \ldots, k,
\end{aligned}
$$

and

$$
\begin{aligned}
c_{j}(x) y^{p_{j}}-\varepsilon_{j} e(x) & \geq-\left[\bar{c}_{j}(x) y^{p_{j}}-\varepsilon_{j}|e(x)|\right] \\
& \geq-\alpha_{j} \bar{c}_{j}^{p / p_{j}}(x)|e(x)|^{\left(p_{i}-p\right) / p_{j}} y^{p}, \quad j=k+1, \ldots, m .
\end{aligned}
$$

Therefore, we can deduce from (2.14) that

$$
\operatorname{div}\left(A(x)\|\nabla y\|^{p-1} \nabla y\right)+\left\langle b(x),\|\nabla y\|^{p-1} \nabla y\right\rangle+C_{2}(x)|y|^{p-1} y \leq 0,
$$

which is the same as (2.4). The remaining argument is the same as in the proof of Theorem 2.1.

Theorem 2.3. Let (A1)-(A3) and (A5) hold. Assume that for any $r \geq$ $r_{0}$, there exist $a_{1}, b_{1}, a_{2}, b_{2}$ such that $r \leq a_{1}<b_{1} \leq a_{2}<b_{2}$, and (2.1) holds. If there exist $u \in \mathcal{D}\left(a_{i}, b_{i}\right), \phi \in \mathbf{C}^{1}\left(\Omega\left(r_{0}\right), \mathbb{R}^{+}\right)$and positive numbers $\delta_{i}, i=1, \ldots, m$, with $\sum_{i=1}^{m} \delta_{i}=1$ such that

$$
\int_{\Omega\left[a_{i}, b_{i}\right]} \phi(x)\left[C_{3}(x) u^{p+1}(\|x\|)-\frac{1}{(p+1)^{p+1}} \frac{|A(x)|^{p+1}}{\lambda_{\min }^{p}(x)}\|H(x)\|^{p+1}\right] d x>0
$$

for $i=1,2$, where $H(x)$ is defined in Theorem 2.1, and

$$
\begin{aligned}
C_{3}(x) & =c(x)+\sum_{i=1}^{m} \alpha_{i} c_{i}^{p / p_{i}}(x)|e(x)|^{\left(p_{i}-p\right) / p_{i}}, \\
\alpha_{i} & =p_{i} p^{-p / p_{i}}\left(p_{i}-p\right)^{\left(p-p_{i}\right) / p_{i}} \delta_{i}^{\left(p_{i}-p\right) / p_{i}},
\end{aligned}
$$

then equation (1.1) is oscillatory. 
Proof. Assume to the contrary that $y=y(x)$ is a nonoscillatory solution and it is eventually positive, say $y(x)>0$ for all $x \in \Omega\left(a_{0}\right), a_{0} \geq r_{0}$. Note that (1.1) can be rewritten as

$$
\begin{aligned}
\operatorname{div}\left(A(x)\|\nabla y\|^{p-1} \nabla y\right) & +\left\langle b(x),\|\nabla y\|^{p-1} \nabla y\right\rangle \\
& +c(x)|y|^{p-1} y+\sum_{i=1}^{m}\left[q_{i}(x) y^{p_{i}}-\delta_{i} e(x)\right] \leq 0 .
\end{aligned}
$$

By Lemma 1.3(1), for $x \in \Omega\left(a_{1}, b_{1}\right)$, we get

$$
\begin{aligned}
c_{i}(x) y^{p_{i}}-\delta_{i} e(x) & =c_{i}(x) y^{p_{i}}+\delta_{i}|e(x)| \\
& \geq \alpha_{i} c_{i}^{p / p_{i}}(x)|e(x)|^{\left(p_{i}-p\right) / p_{i}}, \quad i=1, \ldots, m .
\end{aligned}
$$

Therefore, we can deduce from (2.16) and (2.17) that

$$
\operatorname{div}\left(A(x)\|\nabla y\|^{p-1} \nabla y\right)+\left\langle b(x),\|\nabla y\|^{p-1} \nabla y\right\rangle+C_{3}(x)|y|^{p-1} y \leq 0,
$$

which is the same as (2.4). The remaining argument is the same as in the proof of Theorem 2.1 .

The following Theorems 2.4, 2.5 and 2.6 are variants of Theorems 2.1, 2.2 and 2.3 and present sharper results, but cover the case $0<p \leq 1$.

TheOrem 2.4. Let $0<p \leq 1$, and (A1)-(A4) hold. Assume that for any $r \geq r_{0}$, there exist $a_{1}, b_{1}, a_{2}, b_{2}$ such that $r \leq a_{1}<b_{1} \leq a_{2}<b_{2}$, and (2.1) holds. If there exist $u \in \mathcal{D}\left(a_{i}, b_{i}\right)$ and $\phi \in \mathbf{C}^{1}\left(\Omega\left(r_{0}\right), \mathbb{R}^{+}\right)$such that

$$
\int_{\Omega\left[a_{i}, b_{i}\right]} \phi(x)\left[C_{1}(x) u^{p+1}(\|x\|)-\frac{1}{(p+1)^{p+1}} \lambda_{\max }(x)\|H(x)\|^{p+1}\right] d x>0
$$

for $i=1,2$, where $H(x), C_{1}(x)$ and $\eta_{i}, i=0,1, \ldots, m$, are as in Theorem 2.1, then equation (1.1) is oscillatory.

Proof. Suppose, by contradiction, that (1.1) is nonoscillatory. We start as in the proof of Theorem 2.1 and derive (2.5) which can be written in the form

$$
\begin{aligned}
\operatorname{div} w(x) \leq & -C_{1}(x)-\left\langle b(x) A^{-1}(x), w(x)\right\rangle \\
& -p\left\langle w(x), A^{-1}(x) w(x)\right\rangle\left(\frac{\|\nabla y\|}{|y|}\right)^{1-p} .
\end{aligned}
$$

Note that

$$
\left\langle w(x), A^{-1}(x) w(x)\right\rangle \geq \frac{w^{2}(x)}{\lambda_{\max }(x)}, \quad\|w(x)\| \leq|A(x)|\left(\frac{\|\nabla y\|}{|y|}\right)^{p},
$$

which for $0<p \leq 1$ is equivalent to the inequality

$$
\left(\frac{\|\nabla y\|}{|y|}\right)^{1-p} \geq\left(\frac{\|w(x)\|}{|A(x)|}\right)^{(1-p) / p}=\left(\frac{\|w(x)\|}{\lambda_{\max }(x)}\right)^{(1-p) / p} .
$$


Combining these, we have

$$
\begin{aligned}
\left\langle w(x), A^{-1}(x) w(x)\right\rangle\left(\frac{\|\nabla y\|}{|y|}\right)^{1-p} & \geq \frac{w^{2}(x)}{\lambda_{\max }(x)}\left(\frac{\|w(x)\|}{\lambda_{\max }(x)}\right)^{(1-p) / p} \\
& =\frac{\|w(x)\|^{(p+1) / p}}{\lambda_{\max }^{1 / p}(x)} .
\end{aligned}
$$

Then, substituting (2.20) into (2.19), we get

$$
\operatorname{div} w(x) \leq-C_{1}(x)-\left\langle b(x) A^{-1}(x), w(x)\right\rangle-\frac{p}{\lambda_{\max }^{1 / p}(x)}\|w(x)\|^{(p+1) / p},
$$

which is the same as (2.7). The remaining argument is the same as in the proof of Theorem 2.1.

Combining the techniques of the proofs of Theorems 2.4, 2.2 and 2.3, we have

TheOREm 2.5. Let $0<p \leq 1$ and (A1)-(A4) hold. Assume that for any $r \geq r_{0}$, there exist $a_{1}, b_{1}, a_{2}, b_{2}$ such that $r \leq a_{1}<b_{1} \leq a_{2}<b_{2}$, and (2.12) holds. If there exist $u \in \mathcal{D}\left(a_{i}, b_{i}\right), \phi \in \mathbf{C}^{1}\left(\Omega\left(r_{0}\right), \mathbb{R}^{+}\right)$and positive numbers $\delta_{i}, i=1, \ldots, k$, and $\varepsilon_{j}, j=k+1, \ldots, m$, with $\sum_{i=1}^{k} \delta_{i}+\sum_{j=k+1}^{m} \varepsilon_{j}=1$ such that

$$
\int_{\Omega\left[a_{i}, b_{i}\right]} \phi(x)\left[C_{2}(x) u^{p+1}(\|x\|)-\frac{1}{(p+1)^{p+1}} \lambda_{\max }(x)\|H(x)\|^{p+1}\right] d x>0
$$

for $i=1,2$, where $H(x)$ and $C_{2}(x)$ are defined in Theorem 2.2, then equation (1.1) is oscillatory.

Theorem 2.6. Let $0<p \leq 1$, and (A1)-(A3) and (A5) hold. Assume that for any $r \geq r_{0}$, there exist $a_{1}, b_{1}, a_{2}, b_{2}$ such that $r \leq a_{1}<b_{1} \leq a_{2}<b_{2}$, and (2.1) holds. If there exist $u \in \mathcal{D}\left(a_{i}, b_{i}\right), \phi \in \mathbf{C}^{1}\left(\Omega\left(r_{0}\right), \mathbb{R}^{+}\right)$and positive numbers $\delta_{i}, i=1, \ldots, m$, with $\sum_{i=1}^{m} \delta_{i}=1$ such that

$$
\int_{\Omega\left[a_{i}, b_{i}\right]} \phi(x)\left[C_{3}(x) u^{p+1}(\|x\|)-\frac{1}{(p+1)^{p+1}} \lambda_{\max }(x)\|H(x)\|^{p+1}\right] d x>0
$$

for $i=1,2$, where $H(x)$ and $C_{3}(x)$ are defined in Theorem 2.3 , then equation (1.1) is oscillatory.

Remark 2.1. Let $N=1$ and $\phi(x)=1$. For equation (1.2), Theorems 2.4 and 2.5 reduce to Theorems 1 and 3 in [19], respectively. On the other hand, for (1.1) with $N=1$, Theorem 2.6 reduces to Theorem 2.2 in [31].

REMARK 2.2. Let $m \equiv 1, p=1, b_{i}(x) \equiv 0, c(x) \equiv 0$, and $\phi(x) \equiv 1$ in Theorem 2.4. Note that $p_{1}\left(p_{1}-1\right)^{1 / p_{1}-1}>1$ for $p_{1}>1$, so condition (2.2) in Theorem 2.4 is more general than the condition of Theorem 1 in [32]. 
Therefore, Theorem 2.4 improves and extends the main results in [32]. On the other hand, the method used in [32, starting with integration of the Riccati equality over spheres in $\mathbb{R}^{N}$ centered at the origin, converts the $N$ dimensional problem into a problem in one variable and repeats steps from the proof in [26] to establish the main results. In the present paper, our methodology is somewhat different from [32. We believe that our approach is more natural for partial differential equations and provides a much deeper insight into oscillation. Moreover, our results also take the first order term into account.

REMARK 2.3. Finally, we discuss condition (A4) and pose a problem for future work. Firstly, observe that in condition (A4) (i.e., (1.1) being a mixed type, superlinear and sublinear equation), we cannot allow $k=m$, since otherwise the fact $\sum_{i=1}^{m} p_{i} \eta_{i}>p \sum_{i=1}^{m} \eta_{i}$ (in Lemma 1.1) implies that $\sum_{i=1}^{m} p_{i} \eta_{i} \neq p$, and hence, the conclusions of Theorems 2.2 and 2.4 fail to hold. Secondly, if we let $k=0$ in (A4), i.e., $p>p_{1}>\cdots>p_{m}$, in which case (1.1) is called a mixed sublinear equation, then there is no way to obtain an $m$-tuple $\left(\eta_{1}, \ldots, \eta_{m}\right)$ with $\sum_{i=1}^{m} \eta_{i}<1$ and $0<\eta_{i}<1$ such that $\sum_{i=1}^{m} p_{i} \eta_{i}=p$. Therefore, it is an open problem to find analogous oscillation criteria when $p>p_{1}>\cdots>p_{m}$.

3. Applications. Now we present a method of constructing the test function $u$. Following Philos [16], we say that a function $H(r, s)$ belongs to the function space $\mathcal{H}$ if it is defined on $D=\left\{(r, s): r \geq s \geq r_{0}\right\}$, $H(r, r)=0$ for $r \geq r_{0}$ and $H(r, s)>0$ and has continuous partial derivatives in $D_{0}=\left\{(r, s): r>s \geq r_{0}\right\}$.

For given $H_{1}, H_{2} \in \mathcal{H}$, we define $h_{1}(r, s)$ and $h_{2}(r, s)$ as follows:

$$
\begin{aligned}
& \frac{\partial H_{1}}{\partial r}(r, s)=H_{1}(r, s) h_{1}(r, s), \\
& \frac{\partial H_{2}}{\partial s}(r, s)=H_{2}(r, s) h_{2}(r, s) .
\end{aligned}
$$

Let $u(r)=\left[H_{1}(b, r) H_{2}(r, a)\right]^{1 /(p+1)}$. It is easy to see that $u(b)=u(a)=0$, $u^{p+1}(r)>0$ and $u(r)$ is differentiable on $(a, b)$, with

$$
u^{\prime}(r)=\frac{1}{p+1} u(r)\left[h_{1}(b, r)+h_{2}(r, a)\right] .
$$

In order to make our ideas more transparent, we consider the simple case $m=2$, where (1.1) becomes

$$
\left\{\begin{array}{l}
\operatorname{div}\left(A(x)\|\nabla y\|^{p-1} \nabla y\right)+\left\langle b(x),\|\nabla y\|^{p-1} \nabla y\right\rangle+C(x, y)=e(x), \\
C(x, y)=c(x)|y|^{p-1} y+c_{1}(x)|y|^{p_{1}-1} y+c_{2}(x)|y|^{p_{2}-1} y .
\end{array}\right.
$$

By Theorems 2.1-2.3, we then have 
TheOREM 3.1. Let $p_{1}>p>p_{2}$, let $\eta_{0}, \eta_{1}, \eta_{2}$ be positive constants satisfying

$$
\eta_{0}+\eta_{1}+\eta_{2}=1, \quad p_{1} \eta_{1}+p_{2} \eta_{2}=p,
$$

and let (A1)-(A3) hold. Assume that for any $r \geq r_{0}$, there exist $a_{1}, b_{1}, a_{2}, b_{2}$ such that $r \leq a_{1}<b_{1} \leq a_{2}<b_{2}$ and

$$
\begin{cases}c_{i}(x) \geq 0, & x \in \Omega\left[a_{1}, b_{1}\right] \cup \Omega\left[a_{2}, b_{2}\right], i=1,2, \\ e(x) \leq 0, \quad x \in \Omega\left[a_{1}, b_{1}\right] ; \quad e(x) \geq 0, \quad x \in \Omega\left[a_{2}, b_{2}\right] .\end{cases}
$$

If there exist $H_{1}, H_{2} \in \mathcal{H}$ and $\phi \in \mathbf{C}^{1}\left(\Omega\left(r_{0}\right), \mathbb{R}^{+}\right)$such that $\nabla \phi(x)=$ $\phi(x) b(x) A^{-1}(x)$ and

$$
\begin{array}{r}
\int_{\Omega\left[a_{i}, b_{i}\right]} \phi(x) H_{1}\left(b_{i},\|x\|\right) H_{2}\left(\|x\|, a_{i}\right)\left[\bar{C}_{1}(x)-\frac{1}{(p+1)^{p+1}} \frac{|A(x)|^{p+1}}{\lambda_{\min }^{p}(x)}\right. \\
\left.\times\left|h_{1}\left(b_{i},\|x\|\right)+h_{2}\left(\|x\|, a_{i}\right)\right|^{p+1}\right] d x>0
\end{array}
$$

for $i=1,2$, where

$$
\bar{C}_{1}(x)=c(x)+\left(\prod_{i=0}^{3} \eta_{i}^{-\eta_{i}}\right)|e(x)|^{\eta_{0}} c_{1}^{\eta_{1}}(x) c_{2}^{\eta_{2}}(x),
$$

then equation (3.1) is oscillatory.

REMARK 3.1. $\eta_{0}, \eta_{1}, \eta_{2}$ satisfying condition (3.2) of Theorem 3.1 can indeed be found. For example, $\eta_{1}, \eta_{2}$ may be

$$
\eta_{1}=\frac{p-p_{2}\left(1-\eta_{0}\right)}{p_{1}-p_{2}}, \quad \eta_{2}=\frac{p_{1}\left(1-\eta_{0}\right)-p}{p_{1}-p_{2}},
$$

where $\eta_{0}$ is any positive number satisfying $0<\eta_{0}<\left(p_{1}-p\right) / p_{1}$. This will ensure that (3.2) holds.

Theorem 3.2. Let $p_{1}>p>p_{2}$ and (A1)-(A3) hold. Assume that for any $r \geq r_{0}$, there exist $a_{1}, b_{1}, a_{2}, b_{2}$ such that $r \leq a_{1}<b_{1} \leq a_{2}<b_{2}$ and

$$
\begin{cases}c_{1}(x) \geq 0, & x \in \Omega\left[a_{1}, b_{1}\right] \cup \Omega\left[a_{2}, b_{2}\right], \\ e(x)<0, & x \in \Omega\left(a_{1}, b_{1}\right) ; \quad e(x)>0, \quad x \in \Omega\left(a_{2}, b_{2}\right) .\end{cases}
$$

If there exist $H_{1}, H_{2} \in \mathcal{H}, \phi \in \mathbf{C}^{1}\left(\Omega\left(r_{0}\right), \mathbb{R}^{+}\right)$with $\nabla \phi(x)=\phi(x) b(x) A^{-1}(x)$, and $\delta \in(0,1)$ such that

$$
\begin{array}{r}
\int_{\Omega\left[a_{i}, b_{i}\right]} \phi(x) H_{1}\left(b_{i},\|x\|\right) H_{2}\left(\|x\|, a_{i}\right)\left[\bar{C}_{2}(x)-\frac{1}{(p+1)^{p+1}} \frac{|A(x)|^{p+1}}{\lambda_{\min }^{p}(x)}\right. \\
\left.\times\left|h_{1}\left(b_{i},\|x\|\right)+h_{2}\left(\|x\|, a_{i}\right)\right|^{p+1}\right] d x>0
\end{array}
$$


for $i=1,2$, where

$$
\begin{aligned}
\bar{C}_{2}(x) & =c(x)+\alpha_{1}|e(x)|^{\left(p_{1}-p\right) / p_{1}} c_{1}^{p / p_{1}}(x)-\alpha_{2}|e(x)|^{\left(p_{2}-p\right) / p_{2}} \bar{c}_{2}^{p / p_{2}}(x), \\
\alpha_{1} & =p_{1} p^{-p / p_{1}}\left(p_{1}-p\right)^{\left(p-p_{1}\right) / p_{1}} \delta^{\left(p_{1}-p\right) / p_{1}}, \\
\alpha_{2} & =p_{2} p^{-p / p_{2}}\left(p-p_{2}\right)^{\left(p-p_{2}\right) / p_{2}}(1-\delta)^{\left(p_{2}-p\right) / p_{2}},
\end{aligned}
$$

and $\bar{c}_{2}(x)=\max \left\{-c_{2}(x), 0\right\}$, then equation (3.1) is oscillatory.

Theorem 3.3. Let $p_{1}>p_{2}>p$ and (A1)-(A3) hold. Assume that for any $r \geq r_{0}$, there exist $a_{1}, b_{1}, a_{2}, b_{2}$ such that $r \leq a_{1}<b_{1} \leq a_{2}<b_{2}$ and (3.3) holds. If there exist $H_{1}, H_{2} \in \mathcal{H}$ and $\phi \in \mathbf{C}^{1}\left(\Omega\left(r_{0}\right), \mathbb{R}^{+}\right)$with $\nabla \phi(x)=\phi(x) b(x) A^{-1}(x)$ and $\delta \in(0,1)$ such that

$$
\begin{aligned}
& \int_{\Omega\left[a_{i}, b_{i}\right]} \phi(x) H_{1}\left(b_{i},\|x\|\right) H_{2}(\|\left.x \|, a_{i}\right)\left[\bar{C}_{3}(x)-\frac{1}{(p+1)^{p+1}} \frac{|A(x)|^{p+1}}{\lambda_{\min }^{p}(x)}\right. \\
&\left.\times\left|h_{1}\left(b_{i},\|x\|\right)+h_{2}\left(\|x\|, a_{i}\right)\right|^{p+1}\right] d x>0
\end{aligned}
$$

for $i=1,2$, where

$$
\begin{aligned}
\bar{C}_{3}(x) & =c(x)+\alpha_{1}|e(x)|^{\left(p_{1}-p\right) / p_{1}} c_{1}^{p / p_{1}}(x)+\alpha_{2}|e(x)|^{\left(p_{2}-p\right) / p_{2}} c_{2}^{p / p_{2}}(x), \\
\alpha_{1} & =p_{1} p^{-p / p_{1}}\left(p_{1}-p\right)^{\left(p-p_{1}\right) / p_{1}} \delta^{\left(p_{1}-p\right) / p_{1}}, \\
\alpha_{2} & =p_{2} p^{-p / p_{2}}\left(p_{2}-p\right)^{\left(p-p_{2}\right) / p_{2}}(1-\delta)^{\left(p_{2}-p\right) / p_{2}},
\end{aligned}
$$

then equation (3.1) is oscillatory.

In particular, we shall give the following interesting corollaries. For $\phi \in$ $\mathbf{C}\left(\Omega\left(r_{0}\right), \mathbb{R}^{+}\right)$, define

$$
\lambda(r)=\int_{S(r)} \phi(x) \frac{|A(x)|^{p+1}}{\lambda_{\min }^{p}(x)} d \sigma, \quad \Lambda(r)=\int_{r_{0}}^{r} \frac{d s}{\lambda^{1 / p}(s)},
$$

and recall the Beta function [33,

$$
B(\alpha, \beta)=\int_{0}^{1} s^{\alpha-1}(1-s)^{\beta-1} d s \quad \text { for } \alpha, \beta>0 .
$$

Keep in mind that $B(\alpha, \beta)=B(\beta, \alpha)$.

Corollary 3.1. Let $p_{1}>p>p_{2}$ and $\eta_{0}, \eta_{1}, \eta_{2}$ be positive constants such that (3.2) hold, and let (A1)-(A3) hold. Assume that for any $r \geq r_{0}$, there exist $a_{1}, b_{1}, a_{2}, b_{2}$ such that $r \leq a_{1}<b_{1} \leq a_{2}<b_{2}$ and (3.3) holds. If there exist $\phi \in \mathbf{C}^{1}\left(\Omega\left(r_{0}\right), \mathbb{R}^{+}\right)$with $\nabla \phi(x)=\phi(x) b(x) A^{-1}(x)$ and constants 
$\alpha, \beta>\max \{1, p\}$ such that

$$
\begin{array}{r}
\frac{1}{\left[\Lambda\left(b_{i}\right)-\Lambda\left(a_{i}\right)\right]^{\alpha+\beta-p}} \int_{\Omega\left[a_{i}, b_{i}\right]} \phi(x)\left[\Lambda\left(b_{i}\right)-\Lambda(\|x\|)\right]^{\alpha}\left[\Lambda(\|x\|)-\Lambda\left(a_{i}\right)\right]^{\beta} \bar{C}_{1}(x) d x \\
>\frac{2^{p}}{(p+1)^{p+1}}\left[\alpha^{p+1} B(\alpha-p, \beta+1)+\beta^{p+1} B(\alpha+1, \beta-p)\right]
\end{array}
$$

for $i=1,2$, where $\bar{C}_{1}(x)$ is defined as in Theorem 3.1, then equation (1.1) is oscillatory.

Proof. To apply Theorem 3.1, let

$$
H_{1}\left(b_{i}, s\right)=\left[\Lambda\left(b_{i}\right)-\Lambda(s)\right]^{\alpha}, \quad H_{2}\left(s, a_{i}\right)=\left[\Lambda(s)-\Lambda\left(a_{i}\right)\right]^{\beta} .
$$

Note that

$$
h_{1}\left(b_{i}, s\right)=-\frac{\alpha}{\lambda^{1 / p}(s)\left[\Lambda\left(b_{i}\right)-\Lambda(s)\right]}, \quad h_{2}\left(s, a_{i}\right)=\frac{\beta}{\lambda^{1 / p}(s)\left[\Lambda(s)-\Lambda\left(a_{i}\right)\right]},
$$

and

$$
\begin{aligned}
\int_{\Omega\left[a_{i}, b_{i}\right]} \frac{|A(x)|^{p+1}}{\lambda_{\min }^{p}(x)} \phi(x) H_{1}\left(b_{i},\right. & \|x\|) H_{2}\left(\|x\|, a_{i}\right)\left|h_{1}\left(b_{i},\|x\|\right)+h_{2}\left(\|x\|, a_{i}\right)\right|^{p+1} d x \\
= & \int_{a_{i}}^{b_{i}}\left[\Lambda\left(b_{i}\right)-\Lambda(s)\right]^{\alpha-p-1}\left[\Lambda(s)-\Lambda\left(a_{i}\right)\right]^{\beta-p-1} \\
& \times\left|\alpha\left[\Lambda(s)-\Lambda\left(a_{i}\right)\right]-\beta\left[\Lambda\left(b_{i}\right)-\Lambda(s)\right]\right|^{p+1} d \Lambda(s) .
\end{aligned}
$$

Let $u=\Lambda(s)-\Lambda\left(a_{i}\right)$ and $v=\Lambda\left(b_{i}\right)-\Lambda\left(a_{i}\right)$. Taking into account the elementary inequality

$$
(a+b)^{p+1} \leq 2^{p}\left(a^{p+1}+b^{p+1}\right) \quad \text { for } a, b \geq 0,
$$

we then see that the right-hand side of (3.9) takes the form

$$
\begin{aligned}
& \quad \int_{0}^{v}(v-u)^{\alpha-p-1} u^{\beta-p-1}|\alpha u-\beta(v-u)|^{p+1} d u \\
& \leq 2^{p}\left[\alpha^{p+1} \int_{0}^{v}(v-u)^{\alpha-p-1} u^{\beta} d u+\beta^{p+1} \int_{0}^{v}(v-u)^{\alpha} u^{\beta-p-1} d u\right] \quad(u=v \theta)
\end{aligned}
$$$$
=2^{p} v^{\alpha+\beta-p}\left[\alpha^{p+1} \int_{0}^{1}(1-\theta)^{\alpha-p-1} \theta^{\beta} d \theta+\beta^{p+1} \int_{0}^{1}(1-\theta)^{\alpha} \theta^{\beta-p-1} d \theta\right]
$$$$
=2^{p}\left[\Lambda\left(b_{i}\right)-\Lambda\left(a_{i}\right)\right]^{\alpha+\beta-p}\left[\alpha^{p+1} B(\alpha-p, \beta+1)+\beta^{p+1} B(\alpha+1, \beta-p)\right] .
$$ 
Combining (3.9) and (3.10), we have

$$
\begin{aligned}
& \int_{\Omega\left[a_{i}, b_{i}\right]} \frac{|A(x)|^{p+1}}{\lambda_{\min }^{p}(x)} \phi(x) H_{1}\left(b_{i},\|x\|\right) H_{2}\left(\|x\|, a_{i}\right) \mid h_{1}\left(b_{i},\|x\|+\left.h_{2}\left(\|x\|, a_{i}\right)\right|^{p+1} d x\right. \\
& \leq 2^{p}\left[\Lambda\left(b_{i}\right)-\Lambda\left(a_{i}\right)\right]^{\alpha+\beta-p}\left[\alpha^{p+1} B(\alpha-p, \beta+1)+\beta^{p+1} B(\alpha+1, \beta-p)\right] .
\end{aligned}
$$

From (3.8) and (3.11), we can easily find that (3.4) holds. Hence, by Theorem 3.1 , equation (3.1) is oscillatory.

Similar to the proof of Corollary 3.1, by Theorems 3.2 and 3.3, we have

Corollary 3.2. Let $p_{1}>p>p_{2}$ and (A1)-(A3) hold. Assume that for any $r \geq r_{0}$, there exist $a_{1}, b_{1}, a_{2}, b_{2}$ such that $r \leq a_{1}<b_{1} \leq a_{2}<b_{2}$ and (3.5) holds. If there exist $\phi \in \mathbf{C}^{1}\left(\Omega\left(r_{0}\right), \mathbb{R}^{+}\right)$with $\nabla \phi(x)=\phi(x) b(x) A^{-1}(x)$ and constants $\alpha, \beta>\max \{1, p\}$ and $\delta \in(0,1)$ such that

$$
\begin{array}{r}
\frac{1}{\left[\Lambda\left(b_{i}\right)-\Lambda\left(a_{i}\right)\right]^{\alpha+\beta-p}} \int_{\Omega\left[a_{i}, b_{i}\right]} \phi(x)\left[\Lambda\left(b_{i}\right)-\Lambda(\|x\|)\right]^{\alpha}\left[\Lambda(\|x\|)-\Lambda\left(a_{i}\right)\right]^{\beta} \bar{C}_{2}(x) d x \\
>\frac{2^{p}}{(p+1)^{p+1}}\left[\alpha^{p+1} B(\alpha-p, \beta+1)+\beta^{p+1} B(\alpha+1, \beta-p)\right]
\end{array}
$$

for $i=1,2$, where $\bar{C}_{2}(x)$ is defined as in Theorem 3.2, then equation (3.1) is oscillatory.

COROllary 3.3. Let $p_{1}>p_{2}>p$ and (A1)-(A3) hold. Assume that for any $r \geq r_{0}$, there exist $a_{1}, b_{1}, a_{2}, b_{2}$ such that $r \leq a_{1}<b_{1} \leq a_{2}<b_{2}$ and (3.3) holds. If there exist $\phi \in \mathbf{C}^{1}\left(\Omega\left(r_{0}\right), \mathbb{R}^{+}\right)$with $\nabla \phi(x)=\phi(x) b(x) A^{-1}(x)$ and constants $\alpha, \beta>\max \{1, p\}, \delta \in(0,1)$ such that

$$
\begin{array}{r}
\frac{1}{\left[\Lambda\left(b_{i}\right)-\Lambda\left(a_{i}\right)\right]^{\alpha+\beta-p}} \int_{\Omega\left[a_{i}, b_{i}\right]} \phi(x)\left[\Lambda\left(b_{i}\right)-\Lambda(\|x\|)\right]^{\alpha}\left[\Lambda(\|x\|)-\Lambda\left(a_{i}\right)\right]^{\beta} \bar{C}_{3}(x) d x \\
>\frac{2^{p}}{(p+1)^{p+1}}\left[\alpha^{p+1} B(\alpha-p, \beta+1)+\beta^{p+1} B(\alpha+1, \beta-p)\right]
\end{array}
$$

for $i=1,2$, where $\bar{C}_{3}(x)$ is defined as in Theorem 3.1, then equation (3.1) is oscillatory.

REMARK 3.2. Similar theorems and corollaries can be formulated for Theorems 2.4, 2.5 and 2.6 concerning (3.1). We leave the details to the interested reader.

Finally, we give three examples to illustrate our main results. 
EXAmPle 3.1. Consider equation (3.1) with $N=2$ and

$$
\begin{array}{ll}
A=I \text { (identity matrix), } & b(x)=-\frac{1}{\|x\|^{2}}\left(x_{1}, x_{2}\right), \\
c(x)=c_{0} \sin 2\|x\|, & \\
c_{1}(x)=\frac{3}{5} \sin ^{5 / 3} 2\|x\|, & c_{2}(x)=\frac{9}{25} \cos ^{25 / 9}\|x\|, \\
e(x)=-25 \cos ^{25} 2\|x\|, &
\end{array}
$$

where $x \in \Omega(1), p=3, p_{1}=7 / 2, p_{2}=5 / 2, c_{0} \geq 0$. Let

$$
\eta_{0}=1 / 25, \quad \eta_{1}=3 / 5, \quad \eta_{2}=9 / 25 .
$$

Then $\eta_{0}, \eta_{1}, \eta_{2}$ satisfy $(3.2)$ and

$$
C_{1}(x)=c_{0} \sin 2\|x\|+\frac{1}{2}|\cos 2\|x\|| \sin 2\|x\| .
$$

For any $r \geq 1$, let $a_{1}=2 j \pi, b_{1}=a_{2}=2 j \pi+\pi / 4, b_{2}=2 j \pi+\pi / 2$ for $j=1,2, \ldots$, and $\phi(x)=1 /\|x\|$. Clearly, $\phi(x) b(x) A^{-1}(x)=\nabla \phi(x)$. A direct computation yields

$$
\begin{aligned}
\int_{\Omega\left[a_{i}, b_{i}\right]} \phi(x)\left[C_{1}(x) u^{p+1}(\|x\|)-u^{\prime p+1}(\|x\|)\right] d x \\
=2 \pi \int_{0}^{\pi / 4}\left\{\sin ^{4} 4 s\left[c_{0} \sin 2 s+\frac{1}{4} \sin 4 s\right]-(4 \cos 4 s)^{4}\right\} d s \\
=2 \pi\left[\frac{16}{15} c_{0}+\frac{1}{15}-\frac{3}{2} \pi\right] .
\end{aligned}
$$

Hence, by Theorem 2.1, if we choose the constant $c_{0} \geq 0$ satisfying

$$
c_{0}>\frac{1}{32}(45 \pi-2),
$$

then equation (3.1) with (3.12) is oscillatory.

Example 3.2. Consider equation (3.1) with $N=2$ and

$$
\begin{aligned}
& A=I \text { (identity matrix), } \quad b(x)=-\frac{1}{\|x\|^{2}}\left(x_{1}, x_{2}\right), \\
& c(x)=c_{0} \sin \|x\|, \\
& c_{1}(x)=\sin \|x\|, \quad c_{2}(x)=-\cos \|x\|, \quad e(x)=-\cos \|x\|,
\end{aligned}
$$

where $x \in \Omega(1), p=1, p_{1}=2, p_{2}=1 / 2$ and $c_{0} \geq 0$. For any $r \geq 1$, let $a_{1}=2 j \pi, b_{1}=a_{2}=2 j \pi+\pi / 2, b_{2}=(2 j+1) \pi$ for $j=1,2, \ldots$, and $\delta=1 / 2$. 
A direct computation yields

$C_{2}(x)$

$$
= \begin{cases}c_{0} \sin \|x\|+2^{1 / 2}|\cos \|x\||^{1 / 2} \sin ^{1 / 2}\|x\|-\frac{1}{2} \cos \|x\|, & x \in \Omega\left(a_{1}, b_{1}\right), \\ c_{0} \sin \|x\|+2^{1 / 2} \mid \cos \|x\|^{1 / 2} \sin ^{1 / 2}\|x\|, & x \in \Omega\left(a_{2}, b_{2}\right) .\end{cases}
$$

Let $u(r)=\sin 2 r$ and $\phi(x)=\|x\|$. Clearly, $\phi(x) b(x) A^{-1}(x)=\nabla \phi(x)$. Then

$$
\begin{aligned}
\int_{\Omega\left(a_{1}, b_{1}\right)} & \phi(x)\left[C_{2}(x) u^{2}(\|x\|)-u^{\prime 2}(\|x\|)\right] d x \\
& =2 \pi \int_{0}^{\pi / 2}\left\{\sin ^{2} 2 s\left[c_{0} \sin s+\sin ^{1 / 2} 2 s-\frac{1}{2} \cos s\right]-4 \cos ^{2} 2 s\right\} d s \\
& =2 \pi\left[\frac{8}{15} c_{0}+2^{3 / 2} B\left(\frac{7}{4}, \frac{7}{4}\right)-\frac{4}{15}-\frac{1}{4} \pi\right]
\end{aligned}
$$

and

$$
\begin{aligned}
\int_{\Omega\left(a_{2}, b_{2}\right)} \phi(x)\left[C_{2}(x)\right. & \left.u^{2}(\|x\|)-u^{\prime 2}(\|x\|)\right] d x \\
& =2 \pi \int_{0}^{\pi / 2}\left\{\sin ^{2} 2 s\left[c_{0} \sin s+\sin ^{1 / 2} 2 s\right]-4 \cos ^{2} 2 s\right\} d s \\
& =2 \pi\left[\frac{8}{15} c_{0}+2^{3 / 2} B\left(\frac{7}{4}, \frac{7}{4}\right)-\frac{1}{4} \pi\right] .
\end{aligned}
$$

Hence, by Theorem 2.2, if we choose the constant $c_{0}$ satisfying

$$
\frac{4}{15} c_{0}+2^{1 / 2} B\left(\frac{7}{4}, \frac{7}{4}\right)>\frac{2}{15}+\frac{1}{8} \pi,
$$

then equation (3.1) with (3.13) is oscillatory.

Example 3.3. Consider (3.1) with $N=2$ and

$$
\begin{array}{ll}
A=I \text { (identity matrix), } & b(x)=-\frac{1}{\|x\|^{2}}\left(x_{1}, x_{2}\right), \\
c(x)=c_{0} \sin ^{5 / 3} 2\|x\|, & c_{1}(x)=c_{1}, \quad c_{2}(x)=c_{2}, \\
e(x)=-\cos \|x\|, &
\end{array}
$$

where $x \in \Omega(1), p=1 / 3, p_{1}=1, p_{2}=2 / 3, c_{1} \geq 0, c_{2} \geq 0$. Let $\delta=1 / 3$. Then

$$
C_{3}(x)=c_{0} \sin ^{5 / 3} 2\|x\|+\left(3 c_{1}\right)^{1 / 3}|\cos \|x\||^{2 / 3}+2\left(c_{2} / 3\right)^{1 / 3}|\cos \|x\||^{1 / 2} .
$$

For any $r \geq 1$, let $a_{1}=2 j \pi, b_{1}=a_{2}=2 j \pi+\pi / 2, b_{2}=(2 j+1) \pi$ for $j=1,2, \ldots$. Let $\phi(x)=1 /\|x\|$. Clearly, $\phi(x) b(x) A^{-1}(x)=\nabla \phi(x)$. Let 
$u(r)=\sin 2 r$. We have

$$
\begin{aligned}
& \int_{\Omega\left[a_{i}, b_{i}\right]} \phi(x)\left[C_{3}(x) u^{4 / 3}(\|x\|)-u^{4 / 3}(\|x\|)\right] d x \\
= & 2 \pi \int_{0}^{\pi / 2}\left\{\sin ^{4 / 3} 2 s\left[c_{0} \sin ^{5 / 3} 2 s+\left(3 c_{1}\right)^{1 / 3} \cos ^{2 / 3} s+2\left(c_{2} / 3\right)^{1 / 3} \cos ^{1 / 2} s\right]\right. \\
& \left.-2^{4 / 3} \cos ^{4 / 3} 2 s\right\} d s \\
= & 2 \pi\left[\frac{2}{3} c_{0}+\left(6 c_{1}\right)^{1 / 3} B\left(\frac{7}{6}, \frac{3}{2}\right)+2 c_{2}^{1 / 2} B\left(\frac{7}{6}, \frac{17}{12}\right)-\frac{1}{2} B\left(\frac{7}{6}, \frac{7}{6}\right)\right] .
\end{aligned}
$$

Hence, by Theorem 2.3 if we choose the constants $c_{0}, c_{1}, c_{2}$ satisfying

$$
\frac{2}{3} c_{0}+\left(6 c_{1}\right)^{1 / 3} B\left(\frac{7}{6}, \frac{3}{2}\right)+2 c_{2}^{1 / 2} B\left(\frac{7}{6}, \frac{17}{12}\right)>\frac{1}{2} B\left(\frac{7}{6}, \frac{7}{6}\right),
$$

then equation (3.1) with (3.14) is oscillatory.

Acknowledgments. The author would like to express his great appreciation to the referees for a careful reading and helpful suggestions which led to an improvement of the original manuscript.

\section{References}

[1] D. Çakmak and A. Tiryaki, Oscillation criteria for certain forced second-order nonlinear differential equations, Appl. Math. Lett. 17 (2004), 275-279.

[2] -, - Oscillation criteria for certain forced second-order nonlinear differential equations with delayed argument, Comput. Math. Appl. 49 (2005), 1647-1653.

[3] J. I. Díaz, Nonlinear Partial Differential Equations and Free Boundaries, Vol. I, Elliptic Equations, Pitman Publ., London, 1985.

[4] G. Hardy, J. E. Littewood and G. Pólya, Inequalities, 2nd ed., Cambridge Univ. Press, Cambridge, 1988.

[5] J. Jaroš, T. Kusano and N. Yoshida, Picone-type inequalities for nonlinear elliptic equations and their applications, J. Inequal. Appl. 6 (2001), 387-404.

[6] - - - - - Picone-type inequalities for half-linear elliptic equations and their applications, Adv. Math. Sci. Appl. 12 (2002), 709-724.

[7] - , - - , Picone-type inequalities for nonlinear elliptic equations with first-order terms and their applications, J. Inequal. Appl. 2006, art. ID 52379, 17 pp.

[8] W.-T. Li and R. P. Agarwal, Interval oscillation criteria for a forced second order nonlinear ordinary differential equations, Appl. Anal. 75 (2000), 341-347.

[9] X. Liu and Z. Xu, Oscillation of a forced sup-linear second order differential equation with impulse, Comput. Math. Appl. 53 (2007), 1740-1749.

[10] R. Mař́k, Riccati-type inequality and oscillation criteria for a half-linear PDE with damping, Electron. J. Differential Equations 2004, no. 11, 17 pp. 
[11] R. Mařík, Ordinary differential equations in the oscillation theory of partial halflinear differential equations, J. Math. Anal. Appl. 338 (2008), 194-208.

[12] —, Oscillation Theory of Partial Differential Equations with p-Laplacian, Folia Univ. Agric. et Silvic. Mendel. Brun. Brno, 2008.

[13] A. H. Nasr, Sufficient conditions for the forced super-linear second order differential equations with oscillatory potential, Proc. Amer. Math. Soc. 126 (1998), 123-125.

[14] E. S. Noussair and C. A. Swanson, Oscillation of semilinear elliptic inequalities by Riccati transformation, Canad. J. Math. 32 (1980), 908-923.

[15] M. Picone, Sui valori eccezionali di un parametro da cui dipende un'equazione differenziale lineare ordinaria del second'ordine, Ann. Scuola Norm. Sup. Pisa 11 (1910), 1-141.

[16] Ch. G. Philos, Oscillation theorems for linear differential equations of second order, Arch. Math. (Basel) 53 (1989), 482-492.

[17] Y. G. Sun, A note on Nasr's and Wong's papers, J. Math. Anal. Appl. 286 (2003), 363-367.

[18] Y. G. Sun and F. W. Meng, Interval criteria for oscillation of second order differential equations with mixed nonlinearities, Appl. Math. Comput. 198 (2007), 375-381.

[19] Y. G. Sun and J. S. W. Wong, Oscillation criteria for second order forced ordinary differential equations with mixed nonlinearities, J. Math. Anal. Appl. 334 (2007), 549-560.

[20] A. Tiryaki and Y. Basci, Interval oscillation criteria for second order quasi-linear differential equations, Dynam. Contin. Discr. Impulsive Systems 16 (2009), 233-252.

[21] Q.-R. Wang, Interval criteria for oscillation of second order nonlinear differential equations, J. Comput. Appl. Math. 205 (2007), 231-238.

[22] J. S. W. Wong, Oscillation criteria for a forced second-order linear differential equation, J. Math. Anal. Appl. 231 (1999), 235-240.

[23] Z. Xu, Oscillation criteria for certain damped PDE with p-Laplacian, Glasgow Math. J. 50 (2008), 129-142.

[24] -, Oscillation criteria for damped half-linear PDE via the integral operator, Math. Comput. Modelling. 48 (2008), 1227-1236.

[25] —, Sun-Wong type theorems for second order damped elliptic equations, J. Math. Anal. Appl. 359 (2009), 322-337.

[26] Q. Yang, Interval oscillation criteria for a second order nonlinear ordinary differential equations with oscillatory potential, Appl. Math. Comput. 135 (2003), 49-64.

[27] N. Yoshida, Oscillation of half-linear partial differential equations with first terms, Stud. Univ. Žilina 17 (2003), 177-184.

[28] —, Oscillation criteria for half-linear partial differential equations via Picone's identity, in: Proc. Equadiff 11 (Bratislava, 2005), Bratislava, 2007, 589-598.

[29] -, Oscillation Theory of Partial Differential Equations, World Sci., 2008.

[30] Z. Zheng and F. Meng, Oscillation criteria for second order quasi-linear differential equations, Math. Comput. Modelling 45 (2007), 215-220.

[31] Z. Zheng, X. Wang and H. Han, Oscillation criteria for forced second order differential equations with mixed nonlinearities, Appl. Math. Lett. 22 (2009), 1096-1101.

[32] R.-K. Zhuang, Annual oscillation criteria for second-order nonlinear elliptic differential equations, J. Comput. Anal. Math. 217 (2008), 268-276. 
[33] V. A. Zorich, Mathematical Analysis, Part II, 4th corrected ed., MCCME, Moscow, 2002.

\section{Zhiting $\mathrm{Xu}$}

School of Mathematical Sciences

South China Normal University

Guangzhou, 510631, P.R. China

E-mail: xuzhit@126.com

Received 25.6.2009

and in final form 11.9.2009 